\title{
Philonsorbonne
}

15 | 2021

Année 2020-2021

\section{Le critère de démarcation de Popper et la thèse de Duhem-Quine}

Youri CABOT

\section{(2) OpenEdition}

Journals

Édition électronique

URL : https://journals.openedition.org/philonsorbonne/1642

DOI : 10.4000/philonsorbonne. 1642

ISSN : $2270-7336$

Éditeur

Publications de la Sorbonne

Édition imprimée

Date de publication : 1 janvier 2021

Pagination : 11-29

ISSN : 1255-183X

\section{Référence électronique}

Youri CABOT, «Le critère de démarcation de Popper et la thèse de Duhem-Quine », Philonsorbonne [En ligne], 15 | 2021, mis en ligne le 03 février 2021, consulté le 08 juin 2021. URL : http://

journals.openedition.org/philonsorbonne/1642; DOI : https://doi.org/10.4000/philonsorbonne.1642

(c) Tous droits réservés 


\title{
Le critère de démarcation de Popper et la thèse de Duhem-Quine
}

\author{
Youri CABOT
}

Toute la réflexion philosophique de Popper, y compris ses considérations politiques, s'articule autour du problème de la démarcation, c'est-à-dire de la question de savoir par quel critère les sciences se distinguent des pseudo-sciences. La fameuse thèse de Popper à ce sujet, qui est donc aussi le principe fondamental de toute sa philosophie, est que les sciences sont falsifiables, alors que les pseudo-sciences sont infalsifiables. Autrement dit, la falsifiabilité est non seulement un critère de scientificité, mais aussi le critère par lequel les sciences se distinguent des pseudosciences.

Néanmoins, cette thèse de Popper se heurte à la thèse de Duhem-Quine, qui, sous la forme radicale soutenue par Quine, affirme que les propositions scientifiques, loin d'être falsifiables, peuvent être maintenues quelles que soient les observations effectuées.

On peut toujours maintenir la vérité de n'importe quel énoncé, quelles que soient les circonstances. Il suffit d'effectuer des réajustements radicaux dans d'autres régions du système ${ }^{1}$.

Nous nous proposons ici d'exposer et d'évaluer les différentes approches par lesquelles Popper a tenté de défendre la falsifiabilité des sciences contre la thèse de Duhem-Quine, et notamment sa tentative de

1. W.V.O. Quine, Du point de vue logique, trad. française par C. Alsaleh, B. Ambroise, D. Bonnay, S. Bozon, M. Cozic, S. Laugier, Ph. De Rouilhan et J. Vidal-Rosset, Paris, Vrin, 2003, p. 77. 
situer la falsifiabilité au niveau des théories ${ }^{2}$, tout en examinant si ces réponses permettent également de répondre au problème de la démarcation.

Il nous semble en effet que si le fait de situer la falsifiabilité au niveau des théories permet formellement de garantir la falsifiabilité des sciences en dépit de la thèse de Duhem-Quine, cela retire à la falsifiabilité sa fonction de critère de démarcation, reposant ainsi, sous une forme aggravée, la question de la distinction entre sciences et pseudo-sciences.

\section{Le problème de la démarcation}

Popper explique qu'il en est personnellement venu à s'occuper du problème de la démarcation parce qu'il avait le sentiment que la théorie d'Einstein n'était pas de la même espèce que celles de Marx, de Freud, ou d'Adler, et qu'il éprouvait des difficultés à légitimer ce sentiment ${ }^{3}$. Plus précisément, il lui semblait que tous les critères par lesquels on définissait habituellement les théories scientifiques étaient également satisfaits par ces différentes théories. En effet, toutes ces théories sont des systèmes de propositions cohérents, permettent d'expliquer des phénomènes observables, et s'appuient sur des observations concluantes. Il en déduisit donc qu'il devait $\mathrm{y}$ avoir un autre critère de scientificité, satisfait notamment par la théorie d'Einstein, et plus généralement par les théories physiques, chimiques, astronomiques, biologiques, etc., c'est-à-dire par les sciences, mais pas par les théories de Marx, de Freud ou d'Adler, ni plus généralement par les théories métaphysiques, astrologiques, démonologiques, etc., c'est-àdire par les pseudo-sciences.

Par ailleurs, une telle classification n'implique pas que les conceptions pseudo-scientifiques seraient complètement irrationnelles ou dépourvues de sens ${ }^{4}$. Popper insiste même sur le fait que certaines conceptions métaphysiques ont pu jouer un rôle important dans le développement scientifique $^{5}$. Il cite notamment l'exemple de l'atomisme ${ }^{6}$. Ainsi, les pseudosciences peuvent aussi avoir été des proto-sciences.

Essayons maintenant de reconstruire et de clarifier le problème de la démarcation en faisant abstraction des éléments contingents contenus dans ce récit.

2. K.R. Popper, Les Deux problèmes fondamentaux de la théorie de la connaissance, traduction française par Christian Bonnet, Paris, Hermann, 1999, p. 275.

3. K.R. Popper, Conjectures et réfutations. La croissance du savoir scientifique, traduction française par Michelle Irène et Marc B. de Launay, Paris, Payot, 1985, p. 61.

4. K.R. Popper, La Logique de la découverte scientifique, traduction française par Nicole Thyssen-Rutten et Philippe Devaux, Paris, Payot, 1973, p. 34.

5. Ibid., p. 35.

6 Ibid., p. 283-284. 
Il est conforme aux usages linguistiques de dire que la physique, la chimie, l'astronomie, la biologie, etc., sont des sciences, et de dire que la métaphysique, l'astrologie, la démonologie, etc., ne sont pas des sciences. Ainsi, une définition de la science, c'est-à-dire de la notion courante de science exprimée par l'usage normal du terme «science », devrait consister en une conjonction de critères qui seraient des critères de scientificité, et qui seraient tous satisfaits par la physique, la chimie, l'astronomie, la biologie, etc., mais pas par la métaphysique, l'astrologie, la démonologie, etc. Précisons que, pour reprendre la terminologie classique de la Logique de Port-Royal, il s'agit bien ici d'une définition de chose et pas d'une définition de mot $^{\dagger}$, autrement on pourrait arbitrairement décider de la réponse à ce problème, et il n'y aurait en fait aucun problème. Or l'établissement d'une telle définition présente des difficultés. Plus précisément, il se trouve que beaucoup de critères que satisfont la physique, la chimie, l'astronomie, la biologie, etc., sont également satisfaits par la métaphysique, l'astrologie, la démonologie, etc. Par exemple, selon Popper, l'astrologie ou le marxisme peuvent constituer des systèmes de propositions logiquement cohérents, qui permettent de décrire et de proposer des explications formellement valides de phénomènes observables, tout en s'appuyant sur un grand nombre d'observations concluantes ${ }^{8}$. D'ailleurs, Popper insiste bien sur l'insuffisance de ce dernier critère, qui pourrait sembler décisif à un « inductiviste ». Il soutient même qu'il est en fait très facile de satisfaire ce critère ${ }^{9}$. Cette situation est à l'origine de la notion de pseudo-science, qui se définit autant par la négation de certains critères de scientificité que par la conjonction des autres critères de scientificité, notamment la forme théorique, le pouvoir explicatif, et la présence d'un grand nombre d'observations concluantes. Cette notion doit permettre en premier lieu de regrouper les disciplines comme la métaphysique, l'astrologie, la démonologie, etc., qui, sans être des sciences, peuvent néanmoins être prises pour des sciences si l'on n'est pas suffisamment attentif. Elle peut également permettre de cibler des pratiques non scientifiques qui dérivent d'une origine scientifique.

Nous pouvons alors considérer deux hypothèses. Tout d'abord, on peut supposer que les disciplines que nous qualifions de « sciences » ne satisfont aucun autre critère que ceux qui sont satisfaits par les pseudo-sciences. Dans ce cas, il faudrait en conclure que la notion courante de science est indéfinissable, mais aussi que la notion de pseudo-science est contradictoire, et que la distinction entre science et pseudo-science est vide de sens ou arbitraire. Autrement dit, il ne serait pas possible de légitimer la séparation que nous établissons entre des disciplines comme la physique, la chimie, l'astronomie, la biologie, etc., et d'autres disciplines comme la

7. A. Arnauld \& P. Nicole, La Logique ou l'art de penser, Paris, Gallimard, 1992, p. 79.

8. K.R. Popper, Conjectures et réfutations. La croissance du savoir scientifique, op. cit., p. 62 .

9. K.R. Popper, La Logique de la découverte scientifique, op. cit., p. 271. 
métaphysique, l'astrologie, la démonologie, etc., de telle sorte que ces dernières pourraient légitimement prétendre être des disciplines du même type que les premières. Inversement, on peut soutenir que les disciplines que nous qualifions de « sciences » satisfont au moins un critère de scientificité différent de ceux qui sont satisfaits par les pseudo-sciences. Dans ce cas, un tel critère de scientificité serait un critère de démarcation entre sciences et pseudo-sciences, et légitimerait la distinction que nous effectuons habituellement entre des disciplines comme la physique, la chimie, l'astronomie, la biologie, etc., et des disciplines comme la métaphysique, l'astrologie, la démonologie, etc.

Le problème de la démarcation est donc la question de savoir quels sont les critères de scientificité permettant de distinguer les sciences des pseudosciences, l'enjeu étant de légitimer la distinction que nous faisons entre des disciplines comme la physique, la chimie, l'astronomie, la biologie, etc., et des disciplines comme la métaphysique, l'astrologie, la démonologie, etc.

\section{Le critère de falsifiabilité}

Popper propose de répondre au problème de la démarcation par la thèse selon laquelle les sciences satisfont un critère de falsifiabilité que les pseudosciences ne satisfont pas.

« J'entendais tracer une frontière - aussi bien que faire se pouvait - entre les énoncés ou systèmes d'énoncés des sciences empiriques et tous les autres énoncés, que ceux-ci fussent de nature religieuse, métaphysique ou, tout simplement, pseudo-scientifique. [...] Le critère de réfutabilité apporte en effet une solution à ce problème, puisqu'il spécifie que des énoncés ou des systèmes d'énoncés doivent pouvoir entrer en contradiction avec des observations possibles ou imaginables ${ }^{10} »$.

Popper prend pour exemple notable l'expédition d'Eddington qui a pu observer l'effet de lentille gravitationnelle prévu par la théorie d'Einstein lors de l'éclipse du 29 mai 1919. Si l'on a bien ici un cas d'observation conforme à la prévision, nous avons aussi, et c'est ce qui est le plus important du point de vue épistémologique de Popper, une prévision qui aurait $p u$ ne pas être du tout conforme à l'observation ${ }^{11}$.

Néanmoins, que signifie plus précisément la falsifiabilité ? Dans le passage que nous venons de citer, la falsifiabilité est définie de manière apparemment assez simple : une proposition ou un système de propositions est falsifiable s'il peut entrer en conflit avec des observations possibles ou

10. K.R. Popper, Conjectures et réfutations. La croissance du savoir scientifique, op. cit., p. 68 .

11. Ibid., p. 64 . 
concevables. Néanmoins, cette définition appelle quelques précisions. Tout d'abord, il faut déjouer un contresens malheureusement fréquent, en insistant sur le fait qu'une proposition ou un système de propositions falsifiable ne doit pas nécessairement entrer en conflit avec des observations effectives, ou même disponibles, mais seulement avec des observations possibles ou concevables. Ainsi, si je dis qu'une proposition est falsifiable, je n'affirme ni qu'elle est contredite par des observations effectuées, ni même qu'il existe des états de choses observables qui la contredisent, mais seulement que je peux concevoir un état de choses observable qui la contredirait s'il se produisait. Dans le cas contraire, la falsifiabilité ne serait qu'une forme particulière de fausseté, et la thèse de Popper priverait la science de sa prétention à rechercher la vérité, alors même que Popper affirme que cette prétention est consubstantielle à la science ${ }^{12}$. Ensuite, il faut préciser que la falsifiabilité se traduit par des caractéristiques formelles. Plus précisément, il faut considérer qu'entrer en conflit avec une observation possible ou concevable ne signifie rien de plus qu'être dans une relation de contrariété logique avec une proposition de base. Nous pouvons définir cette dernière notion de manière assez simple, en disant qu'il s'agit de propositions qui entretiennent un rapport privilégié avec l'observation, de telle sorte qu'elles ne peuvent être légitimement tenues pour vraies ou fausses qu'en raison d'une observation particulière. Par exemple, l'affirmation selon laquelle il y a un livre sur mon bureau est une proposition de base. Toutefois, Popper propose une caractérisation plus précise des propositions de base, en soutenant qu'elles doivent être exprimables par des énoncés de la forme « Il $\mathrm{y}$ a $\mathrm{x}$ à l'endroit $\mathrm{k}$ », dans lesquels $\mathrm{x}$ désigne un événement observable ${ }^{13}$. Autrement dit, il s'agit de propositions singulières décrivant des phénomènes observables. Par conséquent, une proposition ou un système de propositions est falsifiable si et seulement s'il est dans un rapport de contrariété logique avec une proposition exprimable par un énoncé de la forme «Il y a x à l'endroit $\mathrm{k} »$ dans lequel $\mathrm{x}$ désigne un événement observable. Par ailleurs, nous pouvons dire, en suivant Popper, qu'une proposition de base qui est en contrariété logique avec une proposition ou un système de proposition, est un falsificateur virtuel de cette proposition ou de ce système de propositions $^{14}$. Enfin, il faut souligner que la falsifiabilité n'est pas simplement la possibilité d'entrer en conflit avec n'importe quelle réalité possible ou concevable, c'est-à-dire d'entretenir un rapport de contrariété logique avec une proposition quelconque. Il s'agirait alors en effet d'une propriété triviale satisfaite par toute proposition, puisque toute proposition est en contradiction logique, et donc a fortiori en contrariété logique, avec sa négation $^{15}$. Or la falsifiabilité consiste à entretenir un rapport de contrariété

12. K.R. Popper, La Logique de la découverte scientifique, op. cit., p. 284.

13. Ibid., p. 101-102.

14. Ibid., p. 84.

15. Il s'agit d'ailleurs d'un critère permettant de distinguer les propositions des pseudopropositions. 
logique non pas avec une proposition quelconque, mais avec une proposition de base. Ainsi, aucun théorème de logique formelle n'est falsifiable, alors que toute proposition a une négation.

Signalons que l'usage popperien du terme «observable» est assez ambigu, puisque Popper souligne que ce terme peut faire l'objet de plusieurs interprétations, et qu'il est même possible de l'employer sans faire aucune référence au processus psychologique de perception ${ }^{16}$. Par exemple, on pourrait considérer que le fait d'être observable se réduit au fait d'avoir certaines dimensions géométriques correspondant au domaine macroscopique. C'est d'ailleurs pourquoi Popper peut affirmer qu'une proposition de base n'est pas la description d'une perception, qui serait susceptible d'être justifiée par l'observation. Il s'agit d'un point important de la philosophie de Popper. Néanmoins, cette question est sans incidence majeure sur notre réflexion actuelle.

Comment la falsifiabilité répond-elle au problème de la démarcation ? Popper ne réduit évidemment pas la scientificité à la falsifiabilité, qui ne peut être que l'un des critères de la scientificité. Toutes les propositions de base sont falsifiables, mais une conjonction de propositions de base ne constituera jamais une science. Il faut donc bien comprendre que le critère de falsifiabilité ne peut servir de critère de démarcation que dans la mesure où il est appliqué à des propositions ou à des systèmes de propositions qui satisfont déjà les autres critères de scientificité, et notamment l'explication de phénomènes observables. Ainsi, le critère de falsifiabilité peut s'appliquer en premier lieu à des propositions conditionnelles universelles, c'est-à-dire des propositions de la forme "Tous les A sont des B », qui permettent justement d'expliquer des phénomènes observables. Précisons que nous présupposons ici que nous ne parlons que de ce que Popper appelle l'universalité au sens strict, qui concerne un nombre illimité d'individus, et non de ce qu'il appelle l'universalité numérique, qui concerne un nombre fini d'individus ${ }^{17}$. Par exemple, si je dis que tous les livres actuellement sur mon bureau sont rouges, il s'agit seulement d'universalité numérique, alors que si je dis que tous les livres sont rouges, il s'agit bien d'universalité au sens strict.

Pour qu'une proposition conditionnelle universelle soit falsifiable, il faut et il suffit qu'il soit possible d'en déduire logiquement une implication testable, c'est-à-dire une proposition de la forme «si p alors q » dans laquelle $\mathrm{p}$ et q sont deux propositions de base. Nous utilisons ici l'expression d'implication testable pour désigner des propositions que Popper qualifie d'énoncés illustratifs ${ }^{18}$ (instantial statement) ou que Hempel qualifie $\mathrm{d}^{\text {'implications vérifiables }}{ }^{19}$. Il nous semble en effet que, comme le remarque

16. Ibid., p. 102.

17. Ibid., p. 60-62.

18 Ibid., p. 100.

19 C.G. Hempel, Éléments d'épistémologie, traduction française par Bertrand Saint-Sernin, Paris, Armand Colin, 2012, p. 31. 
Hempel, le terme «implication» rend bien compte de la nature et de la fonction de ces propositions, mais qu'il est toutefois impropre de les qualifier de «vérifiables». C'est pourquoi nous préférons parler d'implications testables.

Dans ces conditions, il est clair en effet que la conjonction de $\mathrm{p}$ et d'une proposition de base q' incompatible avec q, constitue elle-même une proposition de base $r$ qui est en contrariété logique avec l'implication testable, et donc, par application du modus tollens, avec la proposition conditionnelle universelle de départ. Autrement dit, $r$ est un falsificateur virtuel de cette proposition conditionnelle universelle, qui montre la falsifiabilité de cette dernière.

$$
\begin{aligned}
& \forall \mathrm{x}(\mathrm{Ax} \rightarrow \mathrm{Bx}) \Rightarrow(\mathrm{p} \rightarrow \mathrm{q}) \\
& \mathrm{q}^{\prime} \Rightarrow \neg \mathrm{q} \\
& \mathrm{p} \wedge \mathrm{q}^{\prime}=\mathrm{r} \\
& \mathrm{r} \\
& \Rightarrow(\mathrm{p} \wedge \neg \mathrm{q}) \\
& \Rightarrow \neg(\mathrm{p} \rightarrow \mathrm{q}) \\
& \Rightarrow \neg \forall \mathrm{x}(\mathrm{Ax} \rightarrow \mathrm{Bx})
\end{aligned}
$$

Tous les corbeaux sont noirs

$\Rightarrow$ (cet oiseau est un corbeau $\rightarrow$ cet oiseau est noir)

Cet oiseau est blanc $\Rightarrow$ Cet oiseau n'est pas noir

Cet oiseau est un corbeau $\wedge$ Cet oiseau est blanc

$=$ Cet oiseau est un corbeau non-noir

Cet oiseau est un corbeau non noir

$\Rightarrow$ (Cet oiseau est un corbeau $\wedge$ Cet oiseau n'est pas noir)

$\Rightarrow \neg$ (Cet oiseau est un corbeau $\rightarrow$ Cet oiseau est noir)

$\Rightarrow \neg$ Tous les corbeaux sont noirs

Par conséquent, si nous suivons la thèse de Popper, nous pouvons dire qu'une proposition scientifique est une proposition conditionnelle universelle dont on peut dériver une implication testable, ce qui peut sembler être effectivement le cas des lois scientifiques. Par exemple, la loi galiléenne de la chute libre des corps nous indique qu'un corps lâché en chute libre subit une accélération constante, ce qui permet d'en déduire l'implication testable selon laquelle si nous lâchons un corps en chute libre d'une hauteur $h$ à un instant $t$, ce corps atteindra le sol à un instant $(t+n)$.

La falsifiabilité paraît ainsi n'être que la caractérisation formelle de la testabilité des lois scientifiques. En effet, tester une loi scientifique revient à dériver de celle-ci une implication testable, afin d'observer si, dans les conditions décrites par $\mathrm{p}$, il se produit ou bien l'état de choses décrit par $\mathrm{q}$, ce qui justifie (sans la prouver) l'hypothèse testée, ou bien un état de choses 
décrit par une proposition de base q' incompatible avec q, ce qui réfute l'hypothèse testée. La falsifiabilité est donc aussi bien la possibilité (formelle ou a priori) d'être réfutée par un test.

Cela suggère donc qu'une théorie scientifique est un système de propositions constitué de propositions conditionnelles universelles dont on peut dériver des implications testables, ce qui peut sembler être effectivement le cas des théories scientifiques, puisqu'elles sont constituées de lois scientifiques.

\section{La thèse de Duhem-Quine}

La thèse de Popper est toutefois beaucoup plus discutable que le laisse suggérer la présentation que nous venons d'en faire.

En effet, les implications testables sur lesquelles nous testons effectivement les lois scientifiques, sont souvent dérivées non pas de cette loi considérée isolément, mais de la conjonction de cette loi et d'autres hypothèses, que l'on appelle dans ce contexte des hypothèses auxiliaires.

[...] souvent la dérivation d'une implication vérifiable est moins simple et moins décisive. Prenons, par exemple, l'hypothèse de Semmelweis selon laquelle la fièvre puerpérale est provoquée par un élément infectieux et considérons l'implication vérifiable selon laquelle, si les personnes qui donnent des soins aux patientes se lavent les mains dans une solution de chlorure de chaux, la mortalité due à la fièvre puerpérale sera réduite. Cet énoncé ne découle pas déductivement de la seule hypothèse ; sa dérivation présuppose la prémisse supplémentaire que, à la différence du savon ou de l'eau tout seuls, une solution de chlorure de chaux détruit l'élément infectieux ${ }^{20}$.

Or dans ces conditions, si l'on applique le modus tollens, on constate qu'une proposition de base qui est en contrariété logique avec l'implication testable n'est pas en contrariété logique avec la loi testée, mais avec la conjonction de cette loi et des hypothèses auxiliaires.

$$
\begin{aligned}
& \left(\forall x(A x \rightarrow B x) \wedge H^{\prime} \wedge H^{\prime} \wedge H^{\prime}, \ldots\right) \Rightarrow(p \rightarrow q) \\
& q^{\prime} \Rightarrow \neg q \\
& p^{\prime} \wedge q^{\prime}=r \\
& r \Rightarrow(p \wedge \neg q) \Rightarrow \neg(p \rightarrow q) \Rightarrow \neg\left(\forall x(A x \rightarrow B x) \wedge H^{\prime} \wedge H^{\prime} \wedge H^{\prime}, \ldots\right)
\end{aligned}
$$

20. C.G. Hempel, Éléments d'épistémologie, op. cit., p. 54. 
Par conséquent, une telle proposition de base n'est pas un falsificateur virtuel de la loi testée, et ne montre pas la falsifiabilité de cette dernière. De plus, un test qui s'appuie sur une telle implication testable ne peut plus réfuter la loi testée, mais seulement la conjonction de la loi et des hypothèses auxiliaires, de telle sorte qu'une expérience récalcitrante peut être imputée à l'une des hypothèses auxiliaires présentes dans cette conjonction, ou même à l'absence d'une hypothèse auxiliaire dans cette conjonction, plutôt qu'à la loi testée. On parle alors de holisme de la réfutation. Ainsi, dans l'exemple de Hempel, une expérience récalcitrante permettrait de remettre en cause la loi testée ou, par exemple, l'hypothèse auxiliaire selon laquelle le chlorure de chaux détruit l'élément infectieux, ou même de considérer que le chlorure de chaux qui a été utilisé lors de cette expérience était défectueux.

On peut évidemment être tenté de répondre que le holisme de la réfutation est un phénomène qui est marginal, ou qui peut être évité, et qu'il n'a donc qu'une incidence négligeable sur la thèse de Popper. Pour le dire autrement, on peut supposer qu'il ne décrit qu'une facette accidentelle des lois scientifiques et des tests, qui peut (et qui doit) être évitée. Mais, la thèse de Duhem-Quine soutient justement que le holisme de la réfutation est systématique et inévitable.

Duhem soutient plus précisément que le holisme de la réfutation est systématique et inévitable en physique, ou du moins dans la partie de la physique qui a atteint le stade de la théorie (Duhem évoque en effet à plusieurs reprises des lois de sens commun qui relèvent bien selon lui de la physique, et qui ne sont pas concernées par le holisme de la réfutation. Par exemple: l'éclair précède le tonnerre ${ }^{21}$ ). Il nous semble que son argumentaire peut être reconstruit dans ses grandes lignes par le résumé qui suit. La physique a atteint une forme théorique par l'introduction du formalisme mathématique, et plus précisément du symbolisme arithmétique et numérique ${ }^{22}$. La théorie physique ne peut donc parler que de faits théoriques qui possèdent une précision numérique absolue ${ }^{23}$. Or, les évènements observables sont des faits pratiques qui sont essentiellement imprécis $^{24}$. Je peux percevoir que l'eau est tiède, ou que l'oiseau est sur la branche, mais je ne peux pas percevoir la température de l'eau au degré Celsius près, et je ne peux pas percevoir la position spatiale de l'oiseau au point géométrique près. Par conséquent, à partir des lois physiques, nous ne pouvons pas déduire logiquement quoi que ce soit qui concerne des évènements observables, sans présupposer un système de traduction ${ }^{25}$ entre faits théoriques (c'est-à-dire les états de choses dont peut parler la théorie) et faits pratiques (c'est-à-dire les événements observables). Autrement dit,

21. P. Duhem, La Théorie physique, son objet, sa structure, Paris, Vrin, 2007, p. 234.

22. Ibid., p. 157-158.

23. Ibid., p. 191.

24. Ibid., p. 229.

25. Ibid., p. 190-191. 
on ne peut dériver logiquement une implication testable ${ }^{26}$ à partir d'une loi de physique théorique qu'en présupposant un système de traduction. Ce système de traduction doit notamment s'appuyer sur des instruments de mesure, sur lesquels nous pouvons observer un indicateur nous permettant de lire des valeurs numériques, que nous pouvons ensuite associer à des grandeurs théoriques. Or cette dernière association présuppose elle-même des lois qui proviennent de théories physiques. Par exemple, pour associer le nombre « 28 » que je lis sur mon thermomètre à liquide, à la température en degrés Celsius du corps dans lequel est plongé le thermomètre, je dois présupposer des éléments de thermodynamique concernant la répartition de la chaleur dans les corps et la réaction de certains liquides à la chaleur. Sans quoi, cette association est arbitraire.

On peut certes établir une corrélation entre la chaleur ressentie et le nombre lu sur le thermomètre, mais, sans être interprétée ou traduite, cette corrélation ne signifie pas et ne peut pas signifier que le nombre lu exprime la température en degrés Celsius. Si l'on voulait répondre à cela que l'on pourrait après tout considérer que la température ne signifie rien d'autre que le fait d'être lu sur un thermomètre, on tomberait dans de nombreuses difficultés, car alors on ne pourrait plus comprendre que nous parlons de la même température lorsque nous employons différents instruments de mesure de la température. On trouve une critique d'une telle tentative notamment chez Duhem ${ }^{27}$ et Hempel $^{28}$.

Les arguments de Duhem mènent donc à la conclusion qu'une implication testable d'une loi physique décrit une relation entre des relevés effectués sur des instruments de mesure, et n'est logiquement dérivable de cette théorie physique qu'en présupposant un ensemble d'autres lois physiques, notamment celles qui régissent les instruments de mesure, de telle sorte que, en dernière instance, on ne peut dériver une implication testable d'une loi physique qu'en joignant celle-ci à l'ensemble de la théorie physique. Ainsi, c'est toujours l'ensemble de la théorie physique qui est soumise à un test, et il est donc possible de protéger une loi physique d'une expérience récalcitrante, à condition de modifier d'autres parties de la théorie. Duhem signale l'exemple de Biot, qui a longtemps su protéger l'hypothèse de l'émission en optique des résultats expérimentaux de Fresnel favorables à la théorie ondulatoire ${ }^{29}$.

Par ailleurs, si cet argumentaire concerne la physique, Duhem souligne que de nombreuses disciplines scientifiques emploient des instruments dont le fonctionnement présuppose des éléments de théorie physique ${ }^{30}$. Ainsi, les

26. Bien évidemment, Duhem n'emploie pas cette notion d'implication testable. Nous exposons seulement ici les conséquences des arguments de Duhem dans la terminologie que nous employions précédemment.

27. Ibid., p. 213-215.

28. C.G. Hempel, Éléments d'épistémologie, op. cit., § 7.2.

29. P. Duhem, La Théorie physique, son objet, sa structure, op. cit., p. 302.

30. Ibid., p. 257. 
tests pratiqués dans ces disciplines portent en fait sur la conjonction d'une loi de cette discipline, et de la théorie physique, de telle sorte qu'il s'agit toujours d'un cas de holisme de la réfutation ${ }^{31}$.

Soulignons que si Duhem admet qu'en cas d'expérience récalcitrante, il y a toujours plusieurs interprétations, c'est-à-dire plusieurs ajustements de la théorie, logiquement valables, il soutient également que le bon sens du physicien peut l'aider à savoir quelle est la bonne interprétation ${ }^{32}$. Néanmoins, cette solution ne semble pas pleinement satisfaisante.

Quine défend des positions encore plus radicales. Selon lui, le contenu empirique de n'importe quelle proposition, ce qui renvoie notamment à ses implications testables et ses falsificateurs virtuels, dépend de l'ensemble des propositions scientifiques ${ }^{33}$, et même sans doute de l'ensemble des propositions constituant notre système de croyances $^{34}$. Afin de montrer la radicalité de la position de Quine, considérons l'exemple suivant. Prenons la proposition qui affirme qu'il y a un chat dans cette boîte. Cette proposition ne peut renvoyer à un état de choses perceptible qu'en étant conjointe à un ensemble d'autres propositions. Par exemple, les propositions de zoologie déterminant des caractéristiques visibles du chat, ou les propositions de biologie concernant les facultés de vision de l'observateur, ou les propositions de physique concernant le déplacement de la lumière, etc. Ainsi, si je regarde dans cette boîte et que je ne vois pas de chat, je pourrais considérer que cette expérience invalide des propositions de biologie relatives au chat, ou encore des propositions de physique relatives à la lumière, plutôt que la proposition qui affirme qu'il y a un chat dans cette boîte. D'ailleurs, comme Quine le remarque, il est également toujours possible d'ajouter à notre système de proposition l'affirmation selon laquelle l'observation que nous venons d'effectuer était une hallucination ${ }^{35}$.

Soulignons que Quine lui-même a fortement nuancé sa thèse par la suite $^{36}$. Toutefois, cela ne nous empêche pas nous intéresser ici à la thèse qu'il a présentée dans les « Deux Dogmes de l'empirisme ».

Si l'on considère sérieusement la thèse de Duhem-Quine, il semble difficile de considérer qu'une proposition quelconque, et a fortiori une loi scientifique, puisse être falsifiable. Dans ce cas, peut-on encore distinguer

31. Duhem n'utilise toutefois jamais cet argument pour en conclure que le holisme de la réfutation serait systématique et inévitable dans tous les tests de toutes les sciences.

32. Ibid., p. 301-302.

33. W.V.O. Quine, Du point de vue logique, op. cit., p. 76.

34. Quine ne dirait pas que le contenu empirique d'une proposition dépend d'autres propositions, mais plutôt que l'unité de signification empirique est un ensemble de propositions. Néanmoins, il nous semble que ces deux façons de présenter les choses sont équivalentes.

35. Ibid., p. 77.

36. S.G. Harding (dir.), Can Theories be Refuted? Essays on the Duhem-Quine Thesis, D. Reidel Publishing Company, Dordrecht (Holland), 1976, p. 132. 
les sciences des pseudo-sciences ? Quine lui-même a suggéré que, d'un point de vue logique, la science ne différait pas de la mythologie grecque ${ }^{37}$.

\section{Les réponses de Popper à la thèse de Duhem-Quine}

Popper est revenu à de multiples reprises sur la thèse de Duhem-Quine (étonnamment, on peut remarquer qu'il ne prend pas toujours au sérieux les conséquences de cette thèse, et qu'il lui arrive même de soutenir qu'elle ne pourrait au fond poser de problèmes qu'à une épistémologie vérificationniste, et pas à une épistémologie falsificationniste comme la sienne $^{38}$, alors même que la notion de falsifiabilité est clairement mise en cause par la thèse de Duhem-Quine). Il nous semble que nous pouvons discerner trois approches du problème dans la philosophie de Popper, que nous présentons par ordre croissant de pertinence.

\section{a) Première approche}

Tout d'abord, nous pouvons tenir compte d'une réflexion de Popper qui ne concerne pas spécifiquement la thèse de Duhem-Quine, mais l'attitude consistant à protéger a priori une loi scientifique de toute remise en cause par l'observation, que Popper qualifie d'attitude conventionnaliste ${ }^{39}$.

En résumé, cette attitude conventionnaliste consiste à donner à certaines propositions de la théorie un statut de propositions analytiques, ou plus précisément de définitions verbales. Par exemple, lorsqu'un conventionnaliste (au sens de Popper) dit que tous les cygnes sont blancs, il ne dit pas que tous les individus que nous appelons communément «cygnes » quelle que soit leur couleur, sont blancs, mais que nous ne pouvons utiliser le mot «cygne» que pour des individus qui sont blancs ${ }^{40}$. Ainsi, si on montrait un cygne noir à notre conventionnaliste pour réfuter son affirmation, il pourrait nous répondre que nous confondons le concept courant de cygne qui n'est pas son affaire, avec le concept qu'il exprime par le mot « cygne », qui, par définition, ne peut pas renvoyer à un individu noir.

Popper attribue une telle attitude à Poincaré et à Duhem, mais il nous semble que le cas de Duhem est plus complexe, puisqu'il s'est lui-même opposé à Poincaré. En effet, le holisme de la réfutation de Duhem le pousse à soutenir que toute proposition physique peut éventuellement être remise en cause, et qu'aucune n'a le statut d'axiome ${ }^{41}$.

37. W.V.O. Quine, Du point de vue logique, op. cit., p. 79.

38. K.R. Popper, Conjectures et réfutations. La croissance du savoir scientifique, op. cit., p. 353 .

39. K.R. Popper, La Logique de la découverte scientifique, op. cit., p. 76-79.

40. Autrement dit, les lois du conventionnaliste sont des définitions de mots, plutôt que des définitions de choses.

41. P. Duhem, La Théorie physique, son objet, sa structure, op. cit., p. 298-299. 
Quoi qu'il en soit, Popper admet qu'une telle approche est cohérente et, d'une certaine manière, irréfutable. Plus précisément, être conventionnaliste ou pas est purement affaire de décision ${ }^{42}$. Néanmoins, il reproche au conventionnaliste de contrevenir à des règles méthodologiques qui définissent l'activité scientifique, au même titre que les règles du jeu d'échec définissent la pratique des échecs ${ }^{43}$. Autrement dit, il lui reproche de ne tout simplement pas faire de la science, de ne pas chercher à expliquer les faits, mais de construire simplement un système terminologique. On pourrait reprendre ici un concept de Searle, et dire que le conventionnaliste ne respecte pas les règles constitutives de la science ${ }^{44}$.

La condamnation du conventionnalisme entraîne effectivement l'interdiction de protéger les hypothèses soumises à des tests en modifiant le statut logique de certaines hypothèses auxiliaires (plus précisément en faisant de celles-ci des définitions plutôt que des propositions synthétiques). Par exemple, cela nous interdit de considérer que la vitesse n'est rien d'autre que ce que nous calculons par les lois de notre théorie, afin de protéger une loi mise en cause par un relevé de vitesse contraire aux prévisions. Cela limite donc bien en un sens les possibilités offertes par la thèse de DuhemQuine, mais cela ne suffit pas à résoudre tous les problèmes qu'elle pose. En effet, la thèse de Duhem-Quine permet logiquement de protéger une loi d'une expérience récalcitrante, tout en conservant à toutes les propositions de la théorie leur statut de proposition synthétique.

\section{b) Deuxième approche}

La deuxième approche de Popper consiste tout simplement à nier la thèse de Duhem-Quine, et plus précisément à nier le caractère systématique et inévitable du holisme de la réfutation. Il soutient, exemple à l'appui, qu'il existe des propositions conditionnelles universelles dont on peut logiquement dériver des implications testables sans faire intervenir d'hypothèses auxiliaires, de telle sorte qu'une proposition de base peut logiquement être en contradiction logique avec cette proposition spécifiquement.

[...] si l'on s'interroge sur la falsifiabilité des énoncés universels portant sur la réalité en général, il ne peut y avoir aucun doute que nous sommes justifiés à évaluer négativement sur la base d'expériences au moins certaines propositions universelles simples portant sur la réalité. La proposition : Tous les livres sont reliés en cuir rouge est sans aucun doute une proposition universelle portant sur la réalité et elle est sans aucun doute fausse. Comment a lieu la falsification ? Très simplement : cette proposition liée à l'autre hypothèse : Ceci est un livre fournit les fondements de la déduction de la prédiction : Ce livre est

42. K.R. Popper, La Logique de la découverte scientifique, op. cit., p. 80.

43. Ibid., p. 32.

44. J.R. Searle, La Construction de la réalité sociale, traduction française par Claudine Tiercelin, Paris, Gallimard, 1998. 
relié en cuir rouge. Je peux falsifier cette prédiction. L'une de ses hypothèses doit être fausse. Or la seconde hypothèse était un énoncé particulier portant sur la réalité qui peut être définitivement vérifié. La première hypothèse est donc définitivement falsifiée. Peut-on soulever des objections contre cette considération triviale? Je ne le crois pas : toute objection devrait être dirigée contre la vérifiabilité définitive des énoncés particuliers portant sur la réalité. [...] Que peut-on tirer de l'exemple ? Premièrement, qu'il est en principe possible de falsifier des énoncés universels portant sur la réalité. Des propositions comme Tous les hommes ont les cheveux bruns, Tous les électrons sont visibles à l'œil nu, Qui occupe une fonction en a la compétence, sont précisément sans aucun doute réfutées par des expériences. Deuxièmement, qu'un énoncé universel portant sur la réalité est en tout cas falsifiable lorsque, pour déduire des prédictions, on doit introduire en plus de lui à titre d'hypothèses des énoncés particuliers portant sur la réalité définitivement vérifiables ${ }^{45}$.

On pourrait répondre à cet argument que, d'un point de vue logique, il est indéniable que Popper se trompe. La proposition universelle qui affirme que tous les livres sont reliés en cuir rouge ne permet de prévoir que ceci est un livre relié en cuir rouge, qu'en étant conjointe à la proposition qui affirme que ceci est un livre, ainsi qu'à la proposition selon laquelle l'observateur n'hallucine pas. Il suffit donc de dire que nous sommes en fait en train d'halluciner, pour que notre observation ne permette plus de réfuter la proposition universelle. Toutefois, ce recours à l'argument de l'hallucination est trop radical pour être convaincant, et nous pensons que Popper a raison de ne pas en tenir compte.

Néanmoins, cette réponse à la thèse de Duhem-Quine n'est pas satisfaisante pour autant. En effet, si nous pouvons accorder à Popper que de telles propositions conditionnelles universelles isolément falsifiables existent, il nous semble aussi qu'il s'agit de propositions qui peuvent être aisément réfutées, et qui sont toujours fausses. Prenons une proposition comme celle qui affirme que tous les cygnes sont blancs, qui, prise au pied de la lettre, est bien isolément falsifiable. Cette proposition peut être falsifiée par l'observation d'un cygne peint en noir. De même, la proposition qui affirme que tous les cygnes sont blancs sauf ceux qui sont peints peut être aisément falsifiée par un cygne observé sous un certain éclairage. Et ainsi de suite. Par conséquent, pour que cette proposition ait une chance de ne pas être falsifiée ${ }^{46}$, il nous faut la transformer en une proposition complexe qui comprend une "racine» falsifiable et un ensemble de clauses spécifiques, de telle sorte qu'il suffit de modifier ces clauses pour protéger cette proposition d'une remise en cause par l'observation. Autrement dit, ces

45. K.R. Popper, Les Deux problèmes fondamentaux de la théorie de la connaissance, op. cit., p. 274-275.

46. On pourrait imaginer tout de même des cas de propositions falsifiables de ce type non falsifiées, mais il est certain que la science ne saurait se contenter de telles propositions rares et insuffisantes. 
clauses fonctionnent exactement comme des hypothèses auxiliaires. Lakatos considère ainsi que la fameuse clause ceteris paribus désigne un tel ensemble modulable de clauses fonctionnant comme des hypothèses auxiliaires, et rendant logiquement irréfutable la proposition.

[...] la proposition « tous les cygnes sont blancs », si elle était vraie, serait une simple curiosité, à moins d'affirmer que le fait d'être un cygne cause la blancheur. Mais alors un cygne noir ne réfuterait pas cette proposition, puisqu'il peut seulement indiquer que d'autres causes sont simultanément à l'œuvre. Ainsi, « tous les cygnes sont blancs » est soit une bizarrerie, facile à réfuter, soit une proposition scientifique avec une clause ceteris paribus et par conséquent non réfutable ${ }^{47}$.

Par ailleurs, il n'en reste pas moins des lois scientifiques qui n'ont même pas cette racine falsifiable.

\section{c) Troisième approche}

La troisième approche de Popper consiste à considérer que la thèse de Duhem-Quine n'implique finalement que de resituer la falsifiabilité des sciences au niveau des théories. Plus précisément, les sciences seraient bien falsifiables, au sens où les théories scientifiques, et non les lois scientifiques, seraient falsifiables. En effet, la thèse de Duhem-Quine dit qu'on ne peut déduire une implication testable d'une loi scientifique, et donc qu'on ne peut tester une loi scientifique qu'en présupposant un ensemble d'hypothèses auxiliaires, de telle sorte que l'on peut protéger n'importe quelle partie de la théorie d'une expérience récalcitrante, à condition d'ajuster d'autres parties de la théorie, mais il n'empêche que nous pouvons bien déduire une implication testable de la conjonction de la loi testée et des hypothèses auxiliaires, et que cette conjonction est bel et bien falsifiée en cas d'expérience récalcitrante nous forçant à la modifier. Ainsi, on pourrait simplement ne plus parler de propositions falsifiables, mais seulement de systèmes de propositions, ou de théories falsifiables.

Mais la théorie toute entière, la « conjonction » des hypothèses est en tout cas définitivement falsifiée. Et c'est seulement cela qui importe ${ }^{48}$.

Popper propose d'introduire en ce sens la règle méthodologique suivante :

47. I. Lakatos, Histoire et méthodologie des sciences. Programme de recherche et reconstruction rationnelle, traduction française par Catherine Malamoud et Jean-Fabien Spitz sous la direction de Luce Giard, Paris, PUF, 1994, p. 18.

48. K.R. Popper, Les Deux problèmes fondamentaux de la théorie de la connaissance, op. cit., p. 275. 
[...] l'introduction d'un [nouveau] principe théorique (qui ne peut pas être déduit du système des principes) équivaut à une falsification du système théorique ${ }^{49}$.

D'un point de vue formel, cette réponse permet bien de sauver la falsifiabilité des sciences. Néanmoins, elle pose un sérieux problème, car on ne voit plus très bien en quoi cette falsifiabilité resituée permettrait encore de distinguer les sciences des pseudo-sciences, puisque cela revient à admettre que les sciences peuvent pratiquer les ajustements théoriques successifs que Popper reprochait aux pseudo-sciences comme le marxisme ${ }^{50}$. Autrement dit, la falsifiabilité resituée au niveau des théories ne fonctionne plus comme critère de démarcation formel entre sciences et pseudo-sciences.

\section{Le problème de la démarcation : reprise}

Arrivés à ce stade de la réflexion, il nous semble que nous pouvons établir deux choses importantes. Tout d'abord, si Popper parvient à défendre la falsifiabilité des sciences contre la thèse de Duhem-Quine, c'est au prix de la remise en cause de sa fonction de critère de démarcation. Ensuite, il devient nécessaire de reprendre le problème de la démarcation sous la forme suivante : quels types d'ajustements théoriques à la suite d'une expérience récalcitrante sont caractéristiques de la démarche scientifique (ou interdits dans les sciences)?

À ce sujet, on peut signaler que Popper a essayé d'identifier certaines démarches, notamment dans les ajouts d'hypothèses ad hoc, comme étant typiquement non scientifiques ${ }^{51}$. Mais, il n'est pas évident de trouver un critère satisfaisant permettant de faire le tri entre les différentes hypothèses ad hoc. Même en admettant, à titre de paradigmes, que lorsque Le Verrier conjecture l'existence de Neptune pour expliquer les anomalies du mouvement d'Uranus au regard de la théorie de Newton, il s'agit bien d'un ajustement scientifique, tandis que lorsque des marxistes tentent de protéger la théorie originelle de Marx des démentis flagrants de l'histoire ${ }^{52}$, il ne s'agit pas d'ajustements scientifiques, il reste très difficile de trancher dans de nombreux cas. Par exemple, la tentative de sauver la théorie du

49. Ibid., p. 396.

50. K.R. Popper, Conjectures et réfutations. La croissance du savoir scientifique, op. cit., p. $65-66$.

51. K.R. Popper, Les Deux problèmes fondamentaux de la théorie de la connaissance, op. cit., p. 397-399.

52. Par exemple le fait que, contrairement aux prévisions de Marx, le développement de l'économie capitaliste n'a pas rapidement entraîné la quasi-généralisation de la condition prolétarienne, puisqu'une classe moyenne s'est formée. 
phlogistique des travaux expérimentaux de Lavoisier en faisant l'hypothèse d'un poids négatif du phlogiston constitue-t-elle un ajustement scientifique ou non ${ }^{53}$ ? Par ailleurs, l'ajout d'hypothèses ad hoc n'est pas le seul moyen d'ajuster une théorie à la suite d'une expérience récalcitrante. Nous nous retrouvons finalement avec de très nombreuses façons d'ajuster nos théories face à des expériences récalcitrantes, et on peut donc se demander s'il est vraiment possible d'établir certaines tendances propres à la science (ou interdites dans les sciences).

Néanmoins, nous pensons que les trois conditions du progrès de la connaissance définies par Popper ${ }^{54}$ constituent une piste de réponse prometteuse à notre problème, notamment la troisième condition ${ }^{55}$. Selon celle-ci, une nouvelle théorie ne constitue un progrès pour la connaissance que si elle est soumise avec succès à de nouveaux tests. Plus précisément, il faut que cette théorie soit soumise avec succès à tous les tests auxquels on pouvait soumettre avec succès l'ancienne théorie, mais aussi qu'elle soit soumise à des tests auxquels l'ancienne théorie n'a pas pu être soumise avec succès, ou qui n'avaient jamais été imaginés auparavant. Nous dirons qu'un ajustement théorique est anticipateur s'il satisfait ces conditions de progrès, et réactif dans le cas contraire. Il nous semble que cette condition de progrès peut efficacement être employée dans le cadre de notre problème, pour soutenir qu'un ajustement théorique à la suite d'une expérience récalcitrante est scientifique, s'il s'agit d'un ajustement anticipateur.

Cela impliquerait donc que l'évaluation de la scientificité d'une théorie ne pourrait pas dépendre seulement de caractéristiques formelles, mais aussi $\mathrm{du}$ contexte historique dans lequel cette théorie est produite. Par exemple, reprendre aujourd'hui la théorie de Galilée en ajoutant les hypothèses ad hoc nécessaires pour la rendre compatible avec toutes nos observations astronomiques n'aurait rien de scientifique, alors que cela pouvait l'être au moment où Galilée a produit sa théorie.

\section{Pseudo-science : proto-science et pseudo-mythologie}

Cette dernière remarque nous permet de comprendre que la frontière entre sciences et pseudo-sciences est en tout cas plus fine qu'on ne serait porté à le croire. Le scientifique peut en effet parfaitement préférer un ajustement théorique réactif, et négliger tout ajustement théorique anticipateur. Or une succession de telles décisions éloigne progressivement la théorie de la science, pour la faire dégénérer en pseudo-science (cette notion de dégénérescence est inspirée des processus de déplacements de

53. C.G. Hempel, Éléments d'épistémologie, op. cit., p. 65.

54. K.R. Popper, Conjectures et réfutations. La croissance du savoir scientifique, op. cit., p. 356-367.

55. Ibid., p. 358. 
problèmes dégénératifs de $\operatorname{Lakatos}^{56}$ ). Ainsi, un aristotélicien qui aurait persévéré dans ses positions jusqu'à nos jours au moyen d'ajustements théoriques réactifs aurait progressivement transformé une authentique théorie scientifique en théorie métaphysique pseudo-scientifique, n'ayant pas plus de valeur rationnelle que les discours mythologiques. On pourrait comparer notre aristotélicien fictif au défenseur acharné de la théorie de Newton imaginé par Lakatos ${ }^{57}$ qui tente d'expliquer une anomalie astronomique par la présence d'une planète encore non observée, puis l'impossibilité d'observer cette dernière au moyen d'un télescope par la présence d'un nuage de poussière cosmique, puis l'impossibilité de détecter ce nuage au moyen d'un satellite par la présence d'un champ magnétique, etc.

Une telle dégénérescence aurait d'ailleurs quelque chose de monstrueux, en ce qu'elle serait privée à la fois de toute rationalité scientifique et de tout sens spirituel. Nous ne devrions alors même pas parler d'une mythologie, mais plutôt d'une pseudo-mythologie, qui serait une forme de pseudoscience résultant d'une dégénérescence scientifique, et qui serait privée de la fécondité intellectuelle que peut avoir la pseudo-science quand elle précède la science. La distinction entre science et pseudo-science devrait ainsi être doublée de la distinction entre la pseudo-science proto-scientifique, d'où peut encore surgir de la science, et la pseudo-science pseudo-mythologique, qui est une forme dégénérée de science.

\section{Bibliographie}

ARNAUld, Antoine \& NiCOle, Pierre, La Logique ou l'art de penser, Paris, Gallimard, 1992.

BOYER, Alain, Introduction à la lecture de Karl Popper, Paris, Presses de l'ENS, 1994.

BRENNER, Anastasios, Duhem, science, réalité et apparence, Paris, Vrin, 1990.

DUHEM, Pierre, La Théorie physique, son objet, sa structure, Paris, Vrin, 2007.

HARDING, Sandra G. (dir.), Can Theories be Refuted? Essays on the Duhem-Quine Thesis, D. Reidel Publishing Company, Dordrecht (Holland), 1976.

HEMPEL, Carl Gustav, Éléments d'épistémologie, traduction française par Bertrand Saint-Sernin, Paris, Armand Colin, 2012.

56. I. Lakatos, Histoire et méthodologie des sciences. Programme de recherche et reconstruction rationnelle, op. cit., p. 41.

57. Ibid., p. 14-15. 
LAKatos, Imre, Histoire et méthodologie des sciences. Programme de recherche et reconstruction rationnelle, traduction française par Catherine Malamoud et Jean-Fabien Spitz sous la direction de Luce Giard, Paris, PUF, 1994.

POPPER, Karl Raimund, Les Deux problèmes fondamentaux de la théorie de la connaissance, traduction française par Christian Bonnet, Paris, Hermann, 1999.

POPPER, Karl Raimund, La logique de la découverte scientifique, traduction française par Nicole Thyssen-Rutten et Philippe Devaux, Paris, Payot, 1973.

POPPER, Karl Raimund, Conjectures et réfutations. La croissance du savoir scientifique, trad. française par Michelle Irène et Marc B. de Launay, Paris, Payot, 1985.

QuINE, Willard Van Orman, Du Point de vue logique, traduction française par C. Alsaleh, B. Ambroise, D. Bonnay, S. Bozon, M. Cozic, S. Laugier, Ph. de Rouilhan et J. Vidal-Rosset, Paris, Vrin, 2003.

SEARLE, John R., La Construction de la réalité sociale, traduction française par Claudine Tiercelin, Paris, Gallimard, 1998. 